\title{
Correction to "Fields of Electric Dipoles in Sea Water- The Earth-Atmosphere-Ionosphere Problem"
}

\author{
Wallace L. Anderson
}

Contribution From Electrical Engineering Department, New York University, University Heights 53, N.Y.

(Received August 13, 1962)

\begin{abstract}
A recalculation of the numerical results presented in figure 3 of the paper, "Fields of Electric Dipoles in Sea Water-The Earth-Atmosphere-Ionosphere Problem" has shown that values for the mode solutions, $E 1 \rho^{(v, m)}$ and $E 1 \rho^{(h, m)}$ were in error. Corrected figures show the horizontal to be superior to the vertical dipole over the entire range of 1-1000 $\mathrm{c} / \mathrm{s}$, by virtue of the mode solution.
\end{abstract}

Recent questions raised in a letter to the writer by J. R. Wait concerning the paper "Fields of Electric Dipoles in Sea Water-The Earth-Atmosphere-Ionosphere Problem," Section D, Radio Propagation, of the NBS Journal of Research, 63-72 (Jan.-Feb. 1962) have led to a recalculation of some of the numerical results as presented in figure 3 and commented upon in the text of that paper. The new computations, as made by the writer and checked with independently made computations by Mr. Richard Young at the University of New Mexico Engineering Experiment Station, have shown that originally obtained values of the mode solutions, $E_{1 \rho}^{(v, m)}$ and $E_{1 \rho}^{(h, m)}$, were in error.

Corrected figures showing the variation of $E_{1 \rho}$ with frequency are as follows. For the vertical dipole at $f=1 \mathrm{c} / \mathrm{s}$ :

$$
\begin{gathered}
\left|E_{1 \rho}^{(v, m)}\right|=1.75 \times 10^{-24}\left|M e^{i k_{1}\left(z-z_{0}\right)}\right| \\
\left|E_{1 \rho}^{(v, b)}\right|=4.9 \times 10^{-33}\left|M e^{i k_{1}\left(z-z_{0}\right)}\right| .
\end{gathered}
$$

For the horizontal dipole at $1 \mathrm{c} / \mathrm{s}$ :

$$
\begin{aligned}
& \left|E_{1 \rho}^{(h, m)}\right|=1.1 \times 10^{-17}\left|M \cos \phi e^{i k_{1}\left(z-z_{0}\right)}\right| \\
& \left|E_{1 \rho}^{(h, b)}\right|=4.53 \times 10^{-20}\left|M \cos \phi e^{i k_{1}\left(z-z_{0}\right)}\right| .
\end{aligned}
$$

The corrections do not involve the branch line solutions, which are given above for comparison with the mode solutions. These branch line solutions drop off very rapidly with frequency, as shown in figure 3 of the original paper, and are entirely negligible at $1000 \mathrm{c} / \mathrm{s}$. The mode solutions vary more gradually, and at $1000 \mathrm{c} / \mathrm{s}$ are given by:

$$
\begin{aligned}
& \left|E_{1 \rho}^{(v, m)}\right|=2.9 \times 10^{-19}\left|M e^{i k_{1}\left(z-z_{0}\right)}\right| \\
& \left|E_{1 \rho}^{(h, m)}\right|=2.7 \times 10^{-15}\left|M \cos \phi e^{i k_{1}\left(z-z_{0}\right)}\right| .
\end{aligned}
$$

Accordingly, it is necessary to correct the numbered statements under part 4, "Conclusions." The revised results can be summarized by the remarks:

1. For the range of parameters considered, the horizontal dipole is superior to the vertical one.

2. Under the conditions stated, the branch line contributions can be neglected, since at $f=1 \mathrm{c} / \mathrm{s}$, $\rho=1000 \mathrm{~km}$, the mode solution for the horizontal dipole is greater than the branch line solution by some $47 \mathrm{db}$. It may be pointed out that the factor $e^{-a_{\rho}} / \rho^{2}$ can change by $47 \mathrm{db}$ with a relatively minor change in $\rho$ or frequency, while the Hankel functions are varying rather slowly, at $\rho=1000 \mathrm{~km}$. At closer distances or lower frequencies the branch line solutions should therefore be looked into more closely to evaluate their relative importance. Contributions from more highly damped modes may also be important at closer distances.

3. The system attenuation between half wavelength (in water) "coaxial antennas" separated by $1000 \mathrm{~km}$ is around $205 \mathrm{db}$ at $1 \mathrm{c} / \mathrm{s}$, and around $245 \mathrm{db}$ at $1000 \mathrm{c} / \mathrm{s}$, excluding losses due strictly to propagation through the water. The practicability of such a system for communication purposes therefore depends on magnitude and type of noise, and rate of information transmission.

More details as to corrected numerical results are in preparation as a University of New Mexico Engineering Experiment Station report.

(Paper 67D1-243) 